\begin{tabular}{|c|l|}
\hline Title & Optical Vortices from Liquid Crystal Droplets \\
\hline Author(s) & Brasselet, Etienne; Murazawa, Naoki; Misawa, Hiroaki; Juodkazis, Saulius \\
\hline Citation & $\begin{array}{l}\text { Physical Review Letters, 103(10), 103903 } \\
\text { https://doi.org/10.1103/PhysRevLett.103.103903 }\end{array}$ \\
\hline Issue Date & 2009-09-04 \\
\hline Doc URL & http://hdl.handle.net/2115/39338 \\
\hline Rights & ○ 2009 The A merican Physical Society \\
\hline Type & article \\
\hline File Information & PRL103-10_103903.pdf \\
\hline
\end{tabular}

Instructions for use 


\title{
Optical Vortices from Liquid Crystal Droplets
}

\author{
Etienne Brasselet* \\ Centre de Physique Moléculaire Optique et Hertzienne, CNRS, Université Bordeaux 1, \\ 351 Cours de la Libération, 33405 Talence cedex, France \\ Naoki Murazawa, Hiroaki Misawa, and Saulius Juodkazis \\ Research Institute for Electronic Science, Hokkaido University, N21W10 CRIS Building, Sapporo 001-0021, Japan
}

(Received 25 June 2009; published 2 September 2009)

\begin{abstract}
We report on the generation of mono- and polychromatic optical phase singularities from micron-sized birefringent droplets. This is done experimentally by using liquid crystal droplets whose three dimensional architecture of the optical axis is controlled within the bulk by surfactant agents. Because of its microscopic size these optical vortex generators are optically trapped and manipulated at will, thus realizing a robust self-aligned micro-optical device for orbital angular momentum conversion. Experimental observations are supported by a simple model of optical spin-orbit coupling in uniaxial dielectrics that emphasizes the prominent role of the transverse optical anisotropy with respect to the beam propagation direction.
\end{abstract}

DOI: 10.1103/PhysRevLett.103.103903

Wave singularities in intensity, phase, or polarization, are ubiquitous in nature, being produced when three or more plane waves interfere [1]. Phase singularities are defined as points in space where the phase of the wave is ill-defined and the wave amplitude vanishes-a common feature to all waves [2]. Examples abound in hydrodynamics, low temperature physics and optics. In optics, phase singularities are described by a local azimuthal phase dependence of the electric field of the form $\exp (i \ell \theta)$ where $\ell$ is called the topological charge and $\theta$ is the azimuthal angle. Light beams carrying phase singularities possess a nonzero azimuthal energy flow [3] and are known as optical vortices. Such beams carry nonzero orbital angular momentum [4] and have many applications, which include micromanipulation [5-7], microscopy [8,9], quantum information [10], or astronomical imaging [11].

Optical vortex generation relies on the discontinuous azimuthal reshaping of an initially smooth wave front profile. Optical vortices have been demonstrated for electromagnetic radiation with wavelengths ranging from the millimeter range [12] through to $\mathrm{x}$ rays [13], with much work being performed using visible radiation. Several methods for generating optical vortex beams have been developed. These methods involve physical mechanisms such as refraction [14], diffraction [15], anisotropic Gouy phase manipulation [4] and natural $[16,17]$ or form birefringence [18]. Nowadays, spatial light modulators, which are generally two-dimensional (2D) pixelated liquid crystal (LC) arrays provide an efficient tool for generating vortex beams. Recently, continuously patterned [19-21], 2D liquid crystal devices have also been proposed. However, these millimeter-scale devices are relatively bulky and have a small acceptance angle, preventing widespread use in integrated optics. Approach based on straightforward downsizing of spiral phase plates has
PACS numbers: 42.25.-p, 42.60.Jf, 42.79.-e, 42.81.Gs

been proposed [22], however preventing from handy and versatile uses. The concept of using a spherically symmetric device to generate an optical vortex initially appears somewhat counterintuitive, but, by exploiting the polarization of the incident light and choosing a radially symmetric birefringence, we show that this is possible with a micron scale, self-aligned, omnidirectional optical vortex generator based on a single liquid crystal droplet.

Nematic LCs are uniaxial mesophases having a local orientational order defined by a unit vector $\mathbf{n}$ known as the director. Its orientation can be controlled externally through electrical bias, optical fields, or surface interactions. In this work, we prepared spherical droplets of a controlled micron-order diameter by dispersing the nematic liquid crystal 5CB (4'-n-pentyl-4-cyanobiphenyl, Aldrich) in water. By adding a surfactant (CTAB, cetyltrimethyl-ammonium bromide), the director, and hence the local optical axis, adopt a spherically symmetric 3D spatial distribution in a droplet of a few micrometers in diameter (Fig. 1). This spatial conformation of the optical axis defines an inhomogeneous birefringence that exhibits a defect in the center of the droplet around which the director orientation winds by $2 \pi$. When these "radial droplets" are optically trapped in tightly focused circularly polarized Gaussian laser tweezers (operating at wavelength $\lambda=$ $1.06 \mu \mathrm{m}$ ), (Fig. 1) their natural on-axis positioning in the focal region leads the trapping beam to "read out" the radial birefringence distribution. The generation of a spiraling wave front is thus expected from a geometric Pancharatnam-Berry phase, which arises from the continuous transverse changes of the radial distribution of the optical axis with identical initial and final state all around the center of the droplet [18].

For the purpose of a qualitative demonstration, two droplets were prepared to be close to one another using 


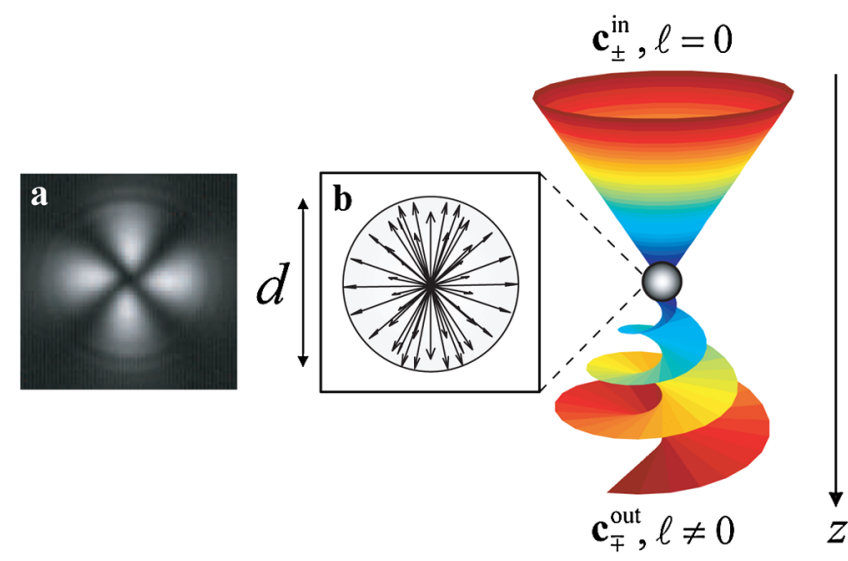

FIG. 1 (color online). Optical vortex generation from a radial nematic liquid crystal droplet. An incident light with circular polarization $\mathbf{c}_{ \pm}$having a smooth wave front profile $(\ell=0)$ is partly converted into the orthogonal polarization state $\mathbf{c}_{\mp}$ that bears a phase singularity $(\ell \neq 0)$. Insets: (a) crossed polarizers image identifying a cylindrically symmetric director distribution and (b) radial structure of the director.

laser manipulation. The $\mathbf{c}_{ \pm}^{\text {out }}$-polarized images of the twin droplets were obtained under spectrally filtered (at $532 \mathrm{~nm}$ ) $\mathbf{c}_{ \pm}^{\text {in }}$-polarized incident white light condenser illumination, where the units vectors $\mathbf{c}_{ \pm}=(\mathbf{x} \pm i \mathbf{y}) / \sqrt{2}$ refer to the circular polarization basis. Therefore, the droplets pair, which behave as twin sources with the same polarization state, give rise to intensity fringes, as shown in Fig. 2. Straight fringes are obtained when the output polarization state is parallel to the input one, $\mathbf{c}^{\text {in }} \cdot \mathbf{c}^{\text {out }}=1$, see Fig. 2(a), whereas curved fringes are evidenced when $\mathbf{c}^{\text {in }} \cdot \mathbf{c}^{\text {out }}=$ 0, as shown in Figs. 2(b) and 2(c). This, respectively, manifests an azimuthally invariant wave front profile [Fig. 2(a)] and a spin-dependent azimuthally varying phase [Figs. 2(b) and 2(c)]. In the latter case, the intensity pattern locally resembles the tilted Young patterns observed in double-slit interferences using optical vortices [23], as shown in the insets of Fig. 2 that display the corresponding table-top double-slit experiments.

It is known that the coherent superposition of two orthogonally polarized light fields produces a total field with space varying polarization. Moreover, the polarization of a beam at any point is determined by the phase difference between the $\mathbf{c}_{ \pm}$components. Assuming the incident polarization to be $\mathbf{c}_{+}^{\text {in }}$, the absolute azimuthal phase structure of

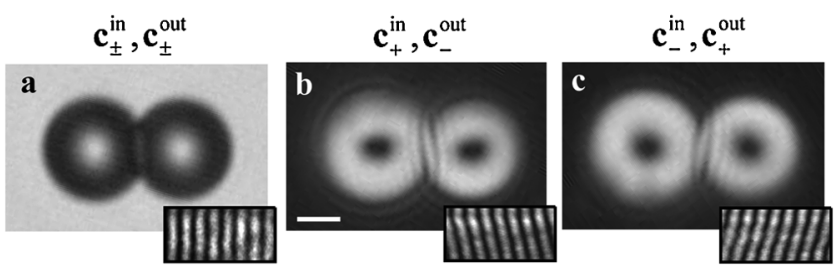

FIG. 2. Twin droplets interferences. Insets: table-top doubleslit Young experiment using a Gaussian beam (a) and a charge two optical vortex [(b) and (c)]. Scale bar is $5 \mu \mathrm{m}$. the $\mathbf{c}_{-}^{\text {out }}$-polarized output field, $\Phi(\theta)$, is therefore quantitatively retrieved from a spatially resolved polarimetric analysis of the output beam [24]. For this purpose, we evaluated the four Stokes parameters $S_{0}=\left|E_{x}\right|^{2}+\left|E_{y}\right|^{2}$, $S_{1}=\left|E_{x}\right|^{2}-\left|E_{y}\right|^{2}, \quad S_{2}=2 \operatorname{Re}\left(E_{x}^{*} E_{y}\right) \quad$ and $\quad S_{3}=$ $2 \operatorname{Im}\left(E_{x}^{*} E_{y}\right)$ of the output beam. Their reduced values $s_{i}=$ $S_{i} / S_{0}(i=1,2,3)$, which all range between -1 and 1 , are shown in Figs. 3(a)-3(c), respectively. By construction, $\Phi(\theta)$ is equal to twice the azimuthal angle of the polarization ellipse of the total output field, $\psi=(1 / 2) \times$ $\arctan \left(s_{2} / s_{1}\right)$, hence $\Phi=2 \psi$. The results are shown in Fig. 4(a) (see markers), which exhibits a uniformly spiraling phase that accumulates a $4 \pi$ phase over a full turn irrespective of the distance from the center of the droplet. Hence, we conclude to the generation of a phase singularity with a topological charge $\ell=2$. To understand the observation of a charge two vortex, one notes that the freely suspended optically trapped droplet is at rest, hence the total light angular momentum (spin + orbital) is conserved. Consequently, the spin angular momentum flipping is associated to the appearance of a $2 \hbar$ orbital angular momentum per photon.

The same system can be used to generate polychromatic vortices by using white light. Panel 1 in Fig. 4(b), shows the characteristic polychromatic doughnut intensity profile. The reddish (blueish) outer (inner) part of the white light doughnut indicates a wavelength-dependent angular momentum conversion process, which merely results from material dispersion [25]. This is emphasized in panels 2, 3, and 4 of Fig. 4(b) where the red, green, and blue components of the vortex exhibit a broader angular spreading at larger wavelengths [26].

The trapping beam itself can be used for in situ and ondemand vortex generation. A unique feature of the proposed technique is that the beam traps the droplets on-axis, and preserves their radial symmetry. Therefore this system enables the generation of on-axis optical vortices without need for the sensitive optical alignment associated with other techniques. For the purpose of demonstration, we trapped droplets with various diameters using beams with various intensities. We then measured the power in each of the circular components of the output beam, and calculated the normalized powers $P_{ \pm} / P_{0}$, where $P_{ \pm}$refers to the output circularly polarized components that are parallel
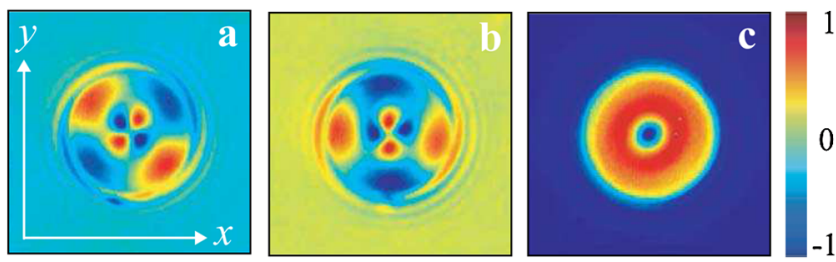

FIG. 3 (color online). Experimental spatially resolved Stokes polarimetry analysis of a single tweezed radial droplet with diameter $\simeq 7 \mu \mathrm{m}$ for quasimonochromatic condenser illumination. (a) $s_{1}$, (b) $s_{2}$, (c) $s_{3}$. 
(a)

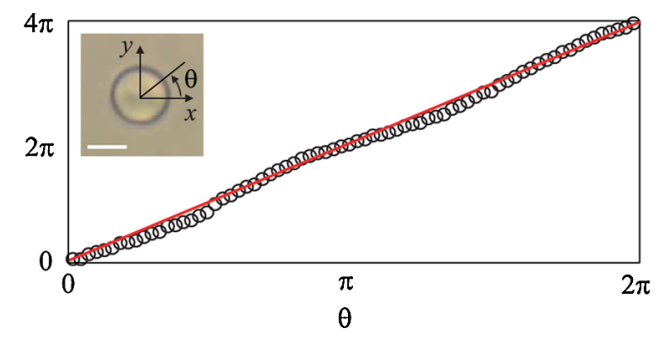

(b)
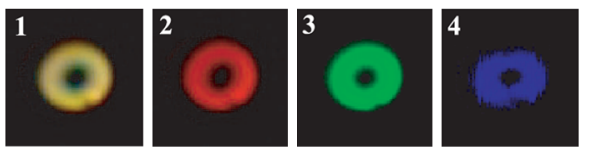

FIG. 4 (color online). (a) Experimental azimuthal phase $\Phi(\theta)$ revealing a phase singularity with charge two $(\ell=2)$. Markers are experimental data and red curve is the theoretical prediction $\Phi(\theta)=2 \theta$. Inset: bright field droplet image, scale bar is $5 \mu \mathrm{m}$. (b) Polychromatic vortex intensity distribution (panel 1) and its RGB components (panels 1, 2, and 3).

$(\ell=0)$ and orthogonal $(\ell=2)$ to the laser tweezers polarization state and $P_{0}$ is equal to the trapping power. We found that the process is almost power independent up to several hundreds of milliwatt but strongly varies with the droplet diameter, as shown in Fig. 5(a). However, the director structure inside the droplet clearly depends on power; see Fig. 5(b). Indeed the droplet image under crossed polarizers exhibits a Maltese cross that becomes more twisted as $P_{0}$ increases, but keeping the axial symmetry and preserving a uniform and continuous winding of the optical axis around the $z$ axis by $2 \pi$ over a full turn.

The dependence of the efficiency of optical vortex generation as a function of droplet diameter is demonstrated in Fig. 5(c). It is found that an increasing amount of the incident Gaussian beam is converted into a vortex as $d$ increases. Although a rigorous theoretical description of the process is expected to be rather complex due to (i) the 3D structure of the inhomogeneous anisotropic droplet and (ii) tight focusing conditions, the observed experimental trend can nevertheless be grasped from simple models in the framework of the paraxial approximation of Gaussian beams and of spin-to-orbital light angular momentum conversion in uniaxial crystals.

For this purpose we first note that the radial droplets possess, on the one hand, the axial symmetry of a $z$-cut uniaxial crystal $[16,17]$ (model 1) and, on the other hand, the radial symmetry of a planar radially patterned birefringent plate [27] (model 2). Optical vortex generation occurs in both geometries with different efficiencies that depend on the material thickness and birefringence. Their respective contributions may, at least qualitatively, be inferred from the knowledge of the averaged contributions of the director along and perpendicular to $z, \mathcal{P}_{\|}=\left\langle\left(\mathbf{n} \cdot \mathbf{e}_{z}\right)^{2}\right\rangle_{\mathcal{S}}$ and $\mathcal{P}_{\perp}=\left\langle\left(\mathbf{n} \times \mathbf{e}_{z}\right)^{2}\right\rangle_{\mathcal{S}}$, respectively, where $\mathbf{e}_{z}$ is the $z$ axis unit vector and $\langle[\ldots]\rangle_{\mathcal{S}}=(1 / 4 \pi) \int_{0}^{2 \pi} \int_{0}^{\pi}[\ldots] \sin \Theta d \Theta d \Phi$ is the averaging over the unit sphere $\mathcal{S}$, with $\Theta$ and $\Phi$ the spherical angles. We obtain $\mathcal{P}_{\|}=1 / 3$ and $\mathcal{P}_{\perp}=2 / 3$. Since these two contributions are of the same order of
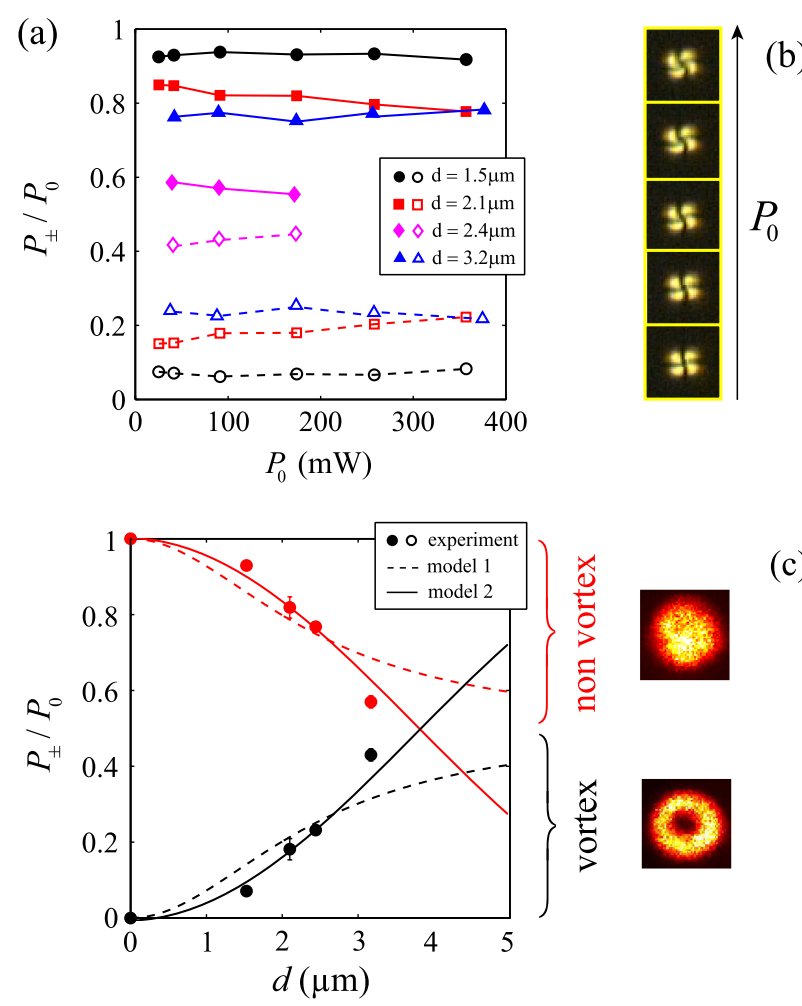

(c)

FIG. 5 (color online). Optical vortex generation for the trapping beam. (a) Fraction of converted, $P_{-} / P_{0}$ (open symbols), and unconverted, $P_{+} / P_{0}$ (filled symbols), photons vs the trapping power $P_{0}$ for different droplet sizes. Circles, squares, triangles and diamonds refer to droplet diameter $d=1.5,2.1$, 2.4, and $3.2 \mu \mathrm{m}$, respectively. (b) Crossed polarized images of the progressively twisted trapped droplet with $d=2.4 \mu \mathrm{m}$ vs power. (c) Power conversion vs $d$ for the vortex (black color) and nonvortex (red color). Markers : experimental data; curves: theoretical prediction from models 1 and 2 (see text for details). Insets: false color intensity distributions of the nonvortex and vortex output beams fields.

magnitude we cannot a priori rule out the use of one model rather than the other and we therefore test two models. The first (second) model comprises an axially homogeneous (radially inhomogeneous) uniaxial slab of thickness $d$, as shown in Fig. 6. The effective birefringence of the slabs, $\tilde{n}_{\|}-\tilde{n}_{\perp}$, with $\tilde{n}_{\|}\left(\tilde{n}_{\perp}\right)$ being the effective refractive indices parallel (perpendicular) to the local optical axis, are found by fitting the experimental data presented in Fig. 5(c).

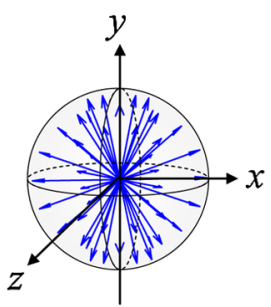

model 1

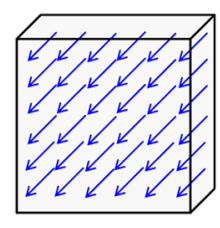

model 2

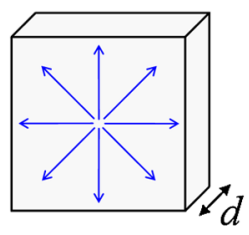

FIG. 6 (color online). Illustration of the director distributions that correspond to model 1 (axial symmetry) and model 2 (radial symmetry). 
Following Refs. [17,25], which deal with circularly polarized Gaussian beams normally incident on a uniaxial crystal having its optical axis along the beam propagation direction (i.e., the $z$ axis), we get for model 1

$$
\left.\frac{P_{ \pm}}{P_{0}}\right|_{\text {model } 1}=\frac{1}{2}\left[1 \pm \frac{1}{1+\left(d / d_{c}\right)^{2}}\right] \text {, }
$$

where $d_{c}=k_{0} w_{0}^{2} \tilde{n}_{\perp} \tilde{n}_{\|}^{2} /\left(\tilde{n}_{\|}^{2}-\tilde{n}_{\perp}^{2}\right)$ is a characteristic power conversion distance and $k_{0}=2 \pi / \lambda, w_{0} \simeq 1.22 \lambda /(2 \mathrm{NA})$ is the beam waist of the trapping beam. For model 2, the fraction of (un)converted power can be obtained in a straightforward manner from the normally incident plane wave situation investigated in Refs. [20,23]. According to these references, the ratio of conversion for an incident plane wave is equal to $\left(P_{ \pm} / P_{0}\right)_{\text {model } 2}=(1 / 2)[1 \pm$ $\cos (2 \pi \Delta \tilde{n} d / \lambda)]$. This result can be expanded to yield the intensity ratio for a Gaussian beam by averaging over the obliquely incident plane waves that constitute the beam and accounting for their individual intensity weights given by $f(\alpha)=\exp \left(-2 \tan ^{2} \alpha / \tan ^{2} \alpha_{0}\right)$ [28], where $\alpha$ refers to the incident angle of a plane wave contribution and $\alpha_{0}$ is the half-divergence angle of the beam, which is taken as $\tan \alpha_{0} \sim w_{0} / z_{0}$, where $z_{0}$ is the Rayleigh distance. We explicitly obtain

$$
\left.\frac{P_{ \pm}}{P_{0}}\right|_{\text {model } 2}=\frac{1}{2}\left[1 \pm\left\langle\cos \left(\frac{2 \pi \Delta \tilde{n}(\alpha) d}{\lambda}\right)\right\rangle_{\alpha}\right],
$$

where $\quad \Delta \tilde{n}(\alpha)=\tilde{n}_{e}(\alpha)-\tilde{n}_{\perp}, \quad$ with $\quad \tilde{n}_{e}(\alpha)=$ $\tilde{n}_{\perp} \tilde{n}_{\|} /\left(\tilde{n}_{\perp}^{2} \cos ^{2} \alpha+\tilde{n}_{\|}^{2} \sin ^{2} \alpha\right)^{1 / 2}$, is the effective anisotropy for the incident angle $\alpha$. Experimental data are independently fitted to models 1 and 2 using Eqs. (1) and (2) respectively with $\tilde{n}_{\|}-\tilde{n}_{\perp}$ being the only fitting parameter. This is made possible by imposing the average effective refractive index, $\left(\tilde{n}_{\perp}+\tilde{n}_{\|}\right) / 2=1.6$, which is the actual average refractive index for the LC. We find $\tilde{n}_{\|}-\tilde{n}_{\perp} \simeq$ 0.91 for the model 1 and $\tilde{n}_{\|}-\tilde{n}_{\perp} \simeq 0.08$ for the model 2 . Although the predictions of model 1 qualitatively fit our observations [see dotted curves, Fig. 5(c)] the unrealistic value obtained for the effective birefringence, i.e., a few times larger than the actual birefringence of the LC $\left(\Delta n_{\mathrm{LC}} \simeq 0.2\right)$, obviously invalidates its use. In contrast, model 2 offers a satisfying agreement with experiments [see solid curves, Fig. 5(c)]. However, the best fit birefringence value that this model yields is roughly twice lower than $\Delta n_{\mathrm{LC}}$. This result can be explained by noting that $\tilde{n}_{\|}-\tilde{n}_{\perp}$ (in model 2) should be associated with the average birefringence of the sphere perpendicular to the $z$ axis, namely $(1 / 4 \pi) \int_{0}^{2 \pi} \int_{0}^{\pi}\left[n_{e}(\Theta)-n_{\perp}\right] d \Theta d \Phi \simeq 0.13$, where $n_{e}(\Theta)=n_{\perp} n_{\|} /\left(n_{\perp}^{2} \cos ^{2} \Theta+n_{\|}^{2} \sin ^{2} \Theta\right)^{1 / 2}$ and $n_{\|} \simeq 1.7$ $\left(n_{\perp} \simeq 1.5\right)$ are the LC refractive indices along and perpendicular to $\mathbf{n}$. Thus we conclude that the transverse optical anisotropy with respect to the beam propagation direction is the main feature governing the optical vortex generator performance.
In this work, we have showed that a liquid droplet of a few microns in diameter can become an optical vortex generator for monochromatic and polychromatic light, by engineering a radial optical axis distribution in a trapped liquid crystal sphere. This offers a simple 3D alternative to expensive 2D multisteps lithography, thus opening the way to the production of dense optical vortex arrays in anisotropic colloids. Together with the advantages of selfalignment and omnidirectional optical incidence, this system could possibly be extended to different spatial distributions of the optical axis, hence permitting the generation of vortices with different topological charges. Moreover, the sensitivity of the liquid crystal molecular ordering to external fields - be it electric, magnetic, thermal, chemical, or the light itself-opens avenues in large-scale array programming and reconfiguration.

This work has been supported by the Institut de Physique Fondamentale de l'Université Bordeaux 1 and Grant-in-Aid No. 19360322 from the Ministry of Education, Science, Sports, and Culture of Japan. We thank O. Wright for careful reading of the manuscript.

*e.brasselet@cpmoh.u-bordeaux1.fr

[1] M. Berry, Nature (London) 403, 21 (2000).

[2] J. F. Nye and M. V. Berry, Proc. R. Soc. A 336, 165 (1974).

[3] J. Arlt, J. Mod. Opt. 50, 1573 (2003).

[4] L. Allen et al., Phys. Rev. A 45, 8185 (1992).

[5] H. He et al., Phys. Rev. Lett. 75, 826 (1995).

[6] K. T. Gahagan and G. A. Swartzlander, Opt. Lett. 21, 827 (1996).

[7] N. B. Simpson et al., Opt. Lett. 22, 52 (1997).

[8] S. W. Hell, Nat. Biotechnol. 21, 1347 (2003).

[9] A. Jesacher et al., Phys. Rev. Lett. 94, 233902 (2005).

[10] G. Molina-Terriza et al., Nature Phys. 3, 305 (2007).

[11] G. Foo et al., Opt. Lett. 30, 3308 (2005).

[12] J. Courtial et al., Phys. Rev. Lett. 80, 3217 (1998).

[13] A. G. Peele et al., Opt. Lett. 27, 1752 (2002).

[14] M. Beijersbergen et al., Opt. Commun. 112, 321 (1994).

[15] N. R. Heckenberg et al., Opt. Lett. 17, 221 (1992).

[16] A. Volyar and T. Fadeyeva, Opt. Spectrosc. 94, 235 (2003).

[17] A. Ciattoni et al., Phys. Rev. E 67, 036618 (2003).

[18] G. Biener et al., Opt. Lett. 27, 1875 (2002).

[19] H. Ren et al., Appl. Phys. Lett. 89, 051114 (2006).

[20] L. Marrucci et al., Phys. Rev. Lett. 96, 163905 (2006).

[21] S. C. McEldowney et al., Opt. Lett. 33, 134 (2008).

[22] G. Knöner et al., Opt. Express 15, 5521 (2007).

[23] H. I. Sztul and R. R. Alfano, Opt. Lett. 31, 999 (2006).

[24] M. S. Soskin et al., Proc. SPIE Int. Soc. Opt. Eng. 5458, 79 (2004).

[25] E. Brasselet et al., Opt. Lett. 34, 1021 (2009).

[26] The wavelength-dependent doughnut prolateness are merely due to nonideal achromatic quarter wave plates.

[27] M. Stalder and M. Schadt, Opt. Lett. 21, 1948 (1996).

[28] The averaging over the angle corresponds to $\langle[\ldots]\rangle_{\alpha}=$ $\int_{0}^{\pi / 2} \sin \alpha f(\alpha)[\ldots] d \alpha / \int_{0}^{\pi / 2} \sin \alpha f(\alpha) d \alpha$. 